\title{
ASO Author Reflections: "Modified Bascule Method" during Robot-Assisted Minimally Invasive Esophagectomy Has Potential to Decrease Recurrent Laryngeal Nerve Palsy
}

\author{
Taro Oshikiri, $\mathrm{MD}^{1}$, and Yoshihiro Kakeji, $\mathrm{MD}^{2}$ \\ ${ }^{1}$ Gastrointestinal Surgery, Kobe University Graduate School of Medicine, Kobe, Japan; ${ }^{2}$ Division of Gastrointestinal \\ Surgery, Department of Surgery, Graduate School of Medicine, Kobe University, Kobe, Japan
}

\section{PAST}

For esophageal squamous cell cancer (ESCC), a few novel techniques for lymphadenectomy along the recurrent laryngeal nerve (RLN) for minimally invasive esophagectomy (MIE) have been developed. ${ }^{1}$ Even though RLN palsy is strongly associated with pneumonia, which is an independent prognostic factor, ${ }^{2,3}$ the rate of RLN palsy remains high.

\section{PRESENT}

Our results revealed that the novel "modified Bascule method" for robot-assisted MIE (RAMIE), based on a logical and simple understanding of the left upper mediastinum anatomy, can promote feasible lymphadenectomy along the left RLN, even when performed during the learning period. ${ }^{4}$

\section{FUTURE}

Larger prospective or retrospective studies are required to validate the findings presented herein. In particular, it should be estimated whether ESCC patients' prognosis will improve due to decrease of pneumonia by this novel procedure for RAMIE.

\section{DISCLOSURE}

Taro Oshikiri and Yoshihiro Kakeji have no conflicts of interest or financial ties to disclose.

\section{REFERENCES}

1. Oshikiri T, Yasuda $\mathrm{T}$, Harada $\mathrm{H}$, et al. A new method (the "Bascule method") for lymphadenectomy along the left recurrent laryngeal nerve during prone esophagectomy for esophageal cancer. Surg Endosc. 2015;29:2442-50.

2. Oshikiri T, Takiguchi G, Hasegawa $\mathrm{H}$, et al. Postoperative recurrent laryngeal nerve palsy is associated with pneumonia in minimally invasive esophagectomy for esophageal cancer. Surg Endosc. 2021;5:837-44.

3. Baba Y, Yoshida N, Shigaki $H$, et al. Prognostic impact of postoperative complications in 502 patients with surgically resected esophageal squamous cell carcinoma: a retrospective single-institution study. Ann Surg. 2016;264:305-11.

4. Oshikiri T, Takiguchi G, Urakawa N, et al. Novel "Modified Bascule method" for lymphadenectomy along the left recurrent laryngeal nerve during robot-assisted minimally invasive esophagectomy. Ann Surg Oncol. 2021. https://doi.org/10.1245/ s10434-021-09738-w.

Publisher's Note Springer Nature remains neutral with regard to jurisdictional claims in published maps and institutional affiliations.

(C) Society of Surgical Oncology 2021

First Received: 8 January 2021

Accepted: 8 January 2021 ;

Published Online: 26 February 2021

T. Oshikiri, MD

e-mail: oshikiri@med.kobe-u.ac.jp 